\title{
WETSEMTM Technology for In-Situ Studies of Dynamic Processes in SEM
}

\author{
Irit Ruach-Nir*, Ofer Zrihan*, Alon Sabban*, Yosi Tzabari* \\ *QuantomiX Ltd, 12 Hamada Street, Tamar Science Park, Rehovot, 76703, Israel
}

Electron microscopy is a very important tool in material science. However, the vacuum environment inside the microscope chamber makes it difficult and in some cases impossible to use in order to follow dynamic processes, such as hydration reactions. Water loss from hydrated specimens cannot be completely avoided even when using the environmental SEM.

The QX-202C capsule (figure 1a) for imaging dynamic processes inside a scanning electron microscope, is a recently developed product by QuantomiX. It is used for direct observation of cementitous systems as well as gypsum systems [1-2]. The WETSEM ${ }^{\mathrm{TM}}$ technology is based on a vacuum tight capsule with an electron transparent window through which the imaging is carried out (figure 1b). The wet sample can be placed and observed in a conventional SEM without the need for drying, freezing or employing low pressure or environmental SEM.

In-situ studies of hydrated systems such as cement or gypsum were carried out inside the SEM applying WETSEM. The wet mixtures were placed in QX-202C sealed specimen capsules specially designed for extended studies inside the microscope chamber. The samples were imaged using a scanning electron microscope operating in high vacuum. The hydration reactions were followed, and images were taken at different stages of the hydration process. Figure 2 shows four images of gypsum, 8 (a), 16 (b), 24 (c), and 60 minutes (d) after mixture preparation. The growth of needle like crystals can be followed in the sequence of micrographs.

Inside the capsules the samples are kept under atmospheric pressure and the "as prepared" water to cement ratio is maintained. Therefore, the hydration process is unaffected by the vacuum in the SEM chamber.

The polymeric membrane through which the imaging is carried out is a few hundreds of nanometers thick and is transparent to x-rays. Therefore, energy dispersive x-ray analysis (EDX) measurements were also employed to identify the chemical composition of the hydration products.

\section{References}

[1] A. Katz, A. Bentur and K. Kovler, submitted to Cement and Concrete Research.

[2] E. Gallucci and K. Scrivener, in preparation. 


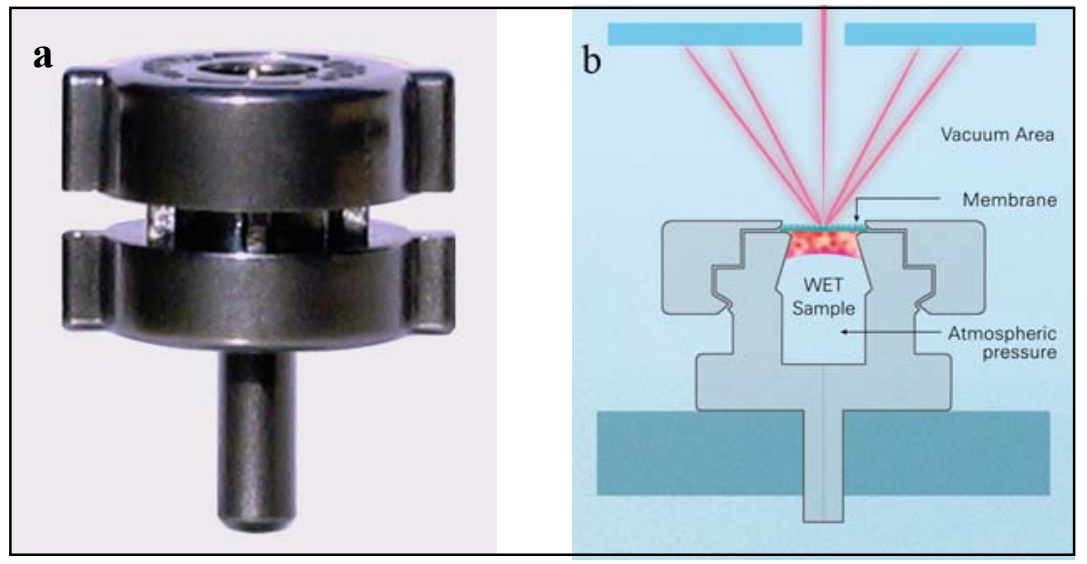

Figure 1. WETSEM technology: Fig. 1a shows picture of QX-202C capsule for dynamic processes. Fig. $1 \mathrm{~b}$ is a schematic representation of WETSEM.
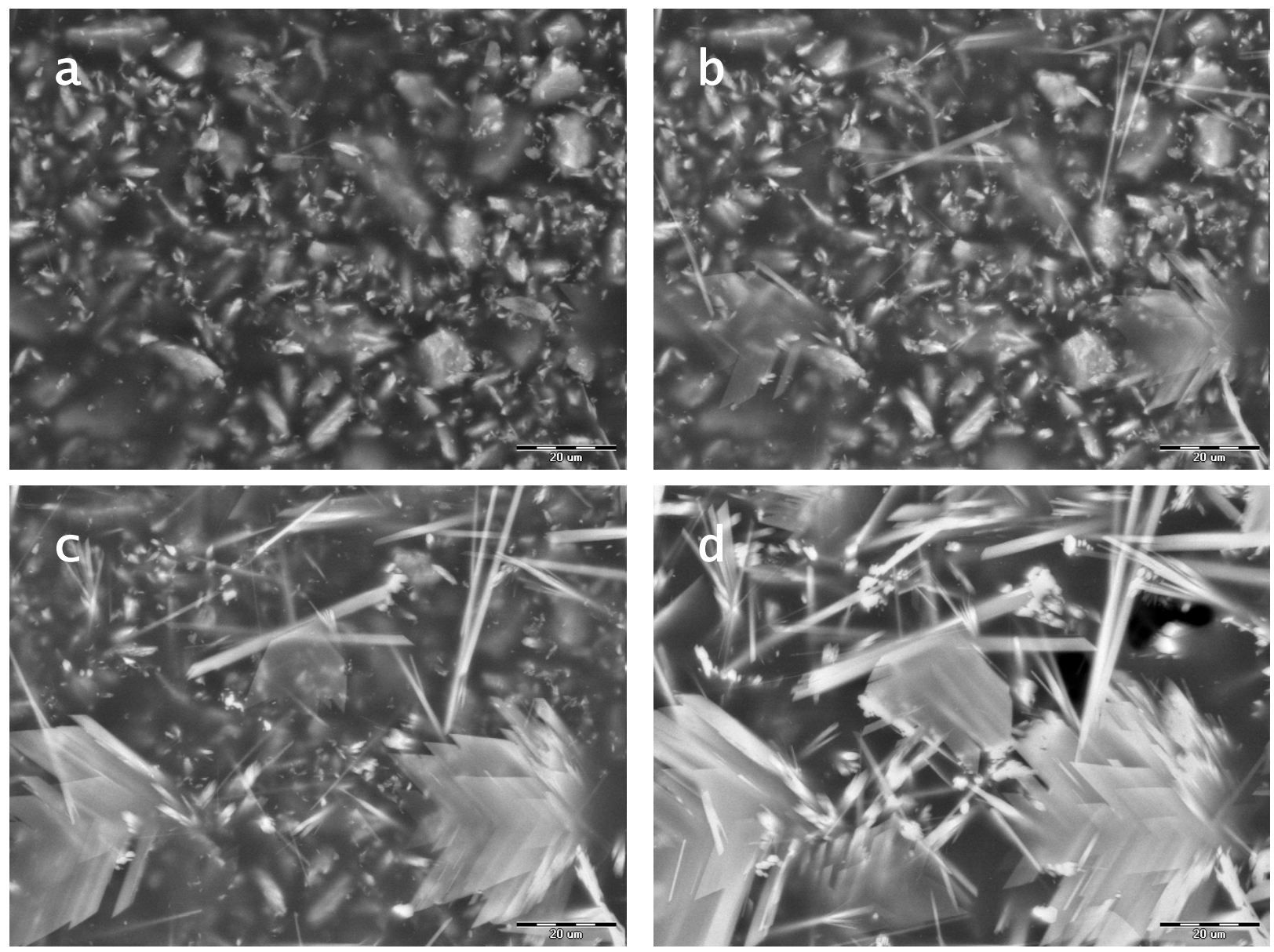

Figure 2. Gypsum hydration at the early stages imaged with a conventional SEM using QX$202 \mathrm{C}$ capsule. Images were taken at 8 (a), 16 (b), 24 (c), and 60 minutes (d). Bar $=20 \mu \mathrm{m}$ 